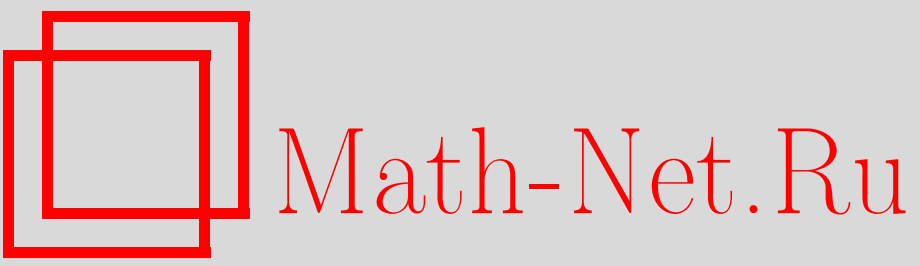

В. Е. Зотеев, М. А. Романюк, А. А. Егорова, Проблема сходимости итерационной процедуры в задаче параметрической идентификации систем с турбулентным трением, Вестн. Сам. гос. техн. ун-та. Сер. Физ.-мат. науки, 2011, выпуск 3(), 108-116

DOI: https://doi.org/10.14498/vsgtu993

Использование Общероссийского математического портала Math-Net.Ru подразумевает, что вы прочитали и согласны с пользовательским соглашением http: //www . mathnet.ru/rus/agreement

Параметры загрузки:

IP : 54.205 .225 .156

26 апреля 2023 г., 14:03:12 
УДК 517.962.24+519.246

\title{
ПРОБЛЕМА СХОДИМОСТИ ИТЕРАЦИОННОЙ ПРОЦЕДУРЫ В ЗАДАЧЕ ПАРАМЕТРИЧЕСКОЙ ИДЕНТИФИКАЦИИ СИСТЕМ С ТУРБУЛЕНТНЫМ ТРЕНИЕМ
}

\author{
В. Е. Зотеев, М. А. Романюк, А. А. Егорова
}

Самарский государственный технический университет, 443100, Самара, ул. Молодогвардейская, 244.

E-mail: zoteev-ve@mail.ru

Рассматривается проблема сходимости итерационной процедурь уточнения коэфбиииентов разностного уравнения в численном методе определения параметров дифференииального оператора для систем с турбулентным трением. Сформулирована и доказана теорема о достаточном условии сходимости итерационной процедуры. При доказательстве теоремы используются свойства сэсимающихся отображений и фундаментальных последователъностей. Как следствие, из теоремы получены бормулы, описывающие априорную и апостериорную оценки погрешности приближений.

Ключевые слова: системь с турбулентным трением, параметрическая идентификация, разностные уравнения, среднеквадратичное приближение.

Достоверная оценка параметров дифференциального оператора на основе статистической обработки результатов наблюдений на выходе системы является важнейшей проблемой в математическом моделировании. Для дифференциальных операторов, описывающих динамические процессы в нелинейных диссипативных системах с турбулентным трением, эта проблема решается на основе стохастических разностных уравнений $[1,2]$. При таком подходе задача сводится к среднеквадратичному оцениванию коэффициентов линейно-параметрической дискретной модели, эффективность которого обеспечивается итерационными процедурами уточнения её коэффициентов [1]. В [2] представлена система стохастических разностных уравнений

$$
\left\{\begin{array}{l}
y_{0}-\frac{1}{\hat{\omega}_{1}^{(p)}} \sin \hat{\omega}^{(p)} t_{0}=-\frac{t_{0}}{\hat{\omega}^{(p)} \tau} \sin \left(\hat{\omega}^{(p)} t_{0}\right) \lambda_{1}+\varepsilon_{0} \\
y_{1}-\frac{1}{\hat{\omega}^{(p)}} \sin \left[\hat{\omega}^{(p)}\left(\tau+t_{0}\right)\right]= \\
=-\left[y_{1}+\frac{t_{0}}{\hat{\omega}^{(p)} \tau} \sin \left[\hat{\omega}^{(p)}\left(\tau+t_{0}\right)\right]\right] \lambda_{1}+\left(1+\lambda_{1}\right) \varepsilon_{1} ; \\
y_{k}+y_{k-2}= \\
=\lambda_{0} y_{k-1}-\lambda_{1}\left[(k-2) y_{k-2}-\lambda_{0}^{(p)}(k-1) y_{k-1}+k y_{k}\right]+\eta_{k} ; \\
\eta_{k}=\left[1+(k-2) \lambda_{1}\right] \varepsilon_{k-2}-\lambda_{0}^{(p)}\left[1+(k-1) \lambda_{1}\right] \varepsilon_{k-1}+\left[1+k \lambda_{1}\right] \varepsilon_{k} ; \\
k=2,3, \ldots, N-1,
\end{array}\right.
$$

описывающая результаты наблюдений $y_{k}$ свободных колебаний системы с турбулентным трением. При среднеквадратичном оценивании коэффициентов

Владимир Евгенъевич Зотеев (д.т.н., доцент), профессор, каф. прикладной математики и информатики. Мария Анатолъевна Романюк, ассистент, каф. прикладной математики и информатики. Александра Арсеновна Егорова, аспирант, каф. прикладной математики и информатики. 
модели (1) используются две вложенные одна в другую итерационные процедуры, одна из которых служит для устранения смещения в оценках [1], а другая предназначена для уточнения оценок $\hat{\lambda}_{0}^{(p)}$ и $\hat{\omega}^{(p)}=\frac{1}{\tau} \arccos \frac{\hat{\lambda}_{0}^{(p)}}{2}, p=$ $=0,1,2, \ldots[2]$. Эта итерационная процедура описывается следующей рекуррентной формулой [2]:

$$
\hat{\lambda}_{0}^{(p)}=(1+c) \hat{\lambda}_{0}^{(p-1)}-c \varphi\left(\hat{\lambda}_{0}^{(p-1)}\right), \quad p=1,2,3, \ldots,
$$

где $\varphi\left(\lambda_{0}\right)$ - непрерывно дифференцируемая функциональная зависимость, определяемая последовательным выполнением известных математических операций, связанных с вычислением и устранением смещения оценки вектора коэффициентов $\lambda=\left(\lambda_{0}, \lambda_{1}\right)^{\top} ; c$ - параметр, величина которого выбирается таким образом, чтобы последовательность приближений $\hat{\lambda}_{0}^{(p)}, p=0,1,2, \ldots$, сходилась при $p \rightarrow \infty$ к решению уравнения $\lambda_{0}=\varphi\left(\lambda_{0}\right)$. Анализ и обеспечение сходимости итерационной процедуры (2) уточнения оценок $\hat{\lambda}_{0}^{(p)}$ и $\hat{\omega}^{(p)}$ является важнейшей задачей при разработке численных методов параметрической идентификации дифференциального оператора для систем с турбулентным трением.

ТеОРема (ДОСТАТочНоЕ УСЛОВИЕ СХодимости). Пусть функиия $\varphi\left(\lambda_{0}\right)$ onpeделена и дифферениируема на отрезке $\left[\lambda_{0}^{(0)}, \lambda_{0}^{(0)}+l\right] \in(-2,2)$, причём $\left|\varphi^{\prime}\left(\lambda_{0}\right)\right| \leqslant$ $\leqslant M, \varphi^{\prime}\left(\lambda_{0}\right) \neq 1$ и выполняется одно из следующих неравенств:

$$
\begin{array}{rll}
0 \leqslant \varphi\left(\lambda_{0}^{(0)}\right)-\lambda_{0}^{(0)} \leqslant(1-M) l & \text { nрu } & 0<M<1, \\
0 \leqslant \operatorname{sign}\left[1-\varphi^{\prime}\left(\lambda_{0}\right)\right]\left[\varphi\left(\lambda_{0}^{(0)}\right)-\lambda_{0}^{(0)}\right] \leqslant m l \quad \text { npu } & \left\{\begin{array}{l}
M>1, \\
\left|\varphi^{\prime}\left(\lambda_{0}\right)-1\right| \geqslant m, \\
0<m<M .
\end{array}\right.
\end{array}
$$

Тогда числовая последовательность $\left\{\lambda_{0}^{(p)}\right\}_{p=0}^{\infty}$, описываемая формулой (2): $\lambda_{0}^{(p)}=(1+c) \lambda_{0}^{(p-1)}-c \varphi\left(\lambda_{0}^{(p-1)}\right)$, будет сходиться независимо от начального приближения $\lambda_{0}^{(0)} \in(-2,2)$ при $p \rightarrow \infty \kappa$ решению уравнения $\lambda_{0}=\varphi\left(\lambda_{0}\right)$ при значениях параметра с, равных

$$
c=\left\{\begin{array}{cll}
-1, & \text { если } & 0<M<1 ; \\
-\operatorname{sign}\left[1-\varphi^{\prime}\left(\lambda_{0}\right)\right] \frac{1}{M+1}, & \text { если } & \left\{\begin{array}{l}
M>1, \\
\left|\varphi^{\prime}\left(\lambda_{0}\right)-1\right| \geqslant m, \\
0<m<M .
\end{array}\right.
\end{array}\right.
$$

Д о ка з а тель с тв в о. Введём следующее обозначение: $\Phi\left(\lambda_{0}\right)=(1+c) \lambda_{0}-$ $-c \varphi\left(\lambda_{0}\right)$. В этом случае итерационная формула (2) принимает вид

$$
\lambda_{0}^{(p)}=\Phi\left(\lambda_{0}^{(p-1)}\right), \quad p=1,2,3, \ldots
$$

Покажем, что последовательность $\left\{\lambda_{0}^{(p)}\right\}_{p=0}^{\infty}$ имеет предел, принадлежащий отрезку $\left[\lambda_{0}^{(0)}, \lambda_{0}^{(0)}+l\right] \in(-2,2)$. В соответствии с (5) для $0<M<1$ имеем 
$c=-1$. При этом из $(2)$ получаем $\Phi\left(\lambda_{0}\right) \equiv \varphi\left(\lambda_{0}\right)$. Отсюда $\left|\Phi^{\prime}\left(\lambda_{0}\right)\right|=\left|\varphi^{\prime}\left(\lambda_{0}\right)\right| \leqslant$ $\leqslant M<1$. При $M>1$ имеем $c=-\frac{1}{1+M}$, если $-M \leqslant \varphi^{\prime}\left(\lambda_{0}\right) \leqslant 1-m<1$ или $c=\frac{1}{1+M}$, если $1<1+m \leqslant \varphi^{\prime}\left(\lambda_{0}\right) \leqslant M$. В первом случае с учётом формулы (2) имеем $\Phi\left(\lambda_{0}\right)=\frac{M}{M+1} \lambda_{0}+\frac{1}{M+1} \varphi\left(\lambda_{0}\right)$ и, соответственно, $\Phi^{\prime}\left(\lambda_{0}\right)=$ $=\frac{M}{M+1}+\frac{1}{M+1} \varphi^{\prime}\left(\lambda_{0}\right)$. Во втором случае, при $1<1+m \leqslant \varphi^{\prime}\left(\lambda_{0}\right) \leqslant M$, получаем $\Phi\left(\lambda_{0}\right)=\frac{M+2}{M+1} \lambda_{0}-\frac{1}{M+1} \varphi\left(\lambda_{0}\right)$ и $\Phi^{\prime}\left(\lambda_{0}\right)=\frac{M+2}{M+1}-\frac{1}{M+1} \varphi^{\prime}\left(\lambda_{0}\right)$. Отсюда с учётом соотношения $0<m<M$ в обоих случаях имеем

$$
\alpha=\max _{\lambda_{0} \in(-2,2)}\left|\Phi^{\prime}\left(\lambda_{0}\right)\right|=\frac{1+M-m}{1+M}<1 .
$$

Используя неравенства (3) или (4), покажем, что последовательность $\left\{\lambda_{0}^{(p)}\right\}_{p=0}^{\infty}$ целиком расположена на отрезке $\left[\lambda_{0}^{(0)}, \lambda_{0}^{(0)}+\rho\right]$, принадлежащем заданному отрезку $\left[\lambda_{0}^{(0)}, \lambda_{0}^{(0)}+l\right]$ :

$$
\lambda_{0}^{(p)} \in\left[\lambda_{0}^{(0)}, \lambda_{0}^{(0)}+\rho\right] \subset\left[\lambda_{0}^{(0)}, \lambda_{0}^{(0)}+l\right], \quad p=0,1,2, \ldots,
$$

где величина $\rho \leqslant l$ обеспечивает выполнение равенства

$$
0 \leqslant \varphi\left(\lambda_{0}^{(0)}\right)-\lambda_{0}^{(0)}=(1-M) \rho
$$

при значениях $M<1$, или выполнение равенства

$$
0 \leqslant \operatorname{sign}\left[1-\varphi^{\prime}\left(\lambda_{0}\right)\right]\left[\varphi\left(\lambda_{0}^{(0)}\right)-\lambda_{0}^{(0)}\right]=m \rho
$$

при $M>1$. Последнее соотношение может быть представлено в виде

$$
\begin{gathered}
0 \leqslant \varphi\left(\lambda_{0}^{(0)}\right)-\lambda_{0}^{(0)}=m \rho=(1-\alpha)(1+M) \rho \text { при }-M \leqslant \varphi^{\prime}\left(\lambda_{0}\right) \leqslant 1-m<1, \\
0 \leqslant \lambda_{0}^{(0)}-\varphi\left(\lambda_{0}^{(0)}\right)=m \rho=(1-\alpha)(1+M) \rho \text { при } 1<1+m \leqslant \varphi^{\prime}\left(\lambda_{0}\right) \leqslant M .
\end{gathered}
$$

Очевидно, что функция $\Phi\left(\lambda_{0}\right)$ непрерывно дифференцируема на отрезке $\left[\lambda_{0}^{(0)}, \lambda_{0}^{(0)}+\rho\right]$, следовательно, для любых $\lambda_{0}^{(p)}, \lambda_{0}^{(q)} \in\left[\lambda_{0}^{(0)}, \lambda_{0}^{(0)}+\rho\right]$ она удовлетворяет условию Липшица:

$$
\left|\Phi\left(\lambda_{0}^{(p)}\right)-\Phi\left(\lambda_{0}^{(q)}\right)\right| \leqslant \alpha\left|\lambda_{0}^{(p)}-\lambda_{0}^{(q)}\right|, \quad \alpha=\max _{\left[\lambda_{0}^{(0)}, \lambda_{0}^{(0)}+\rho\right]}\left|\Phi^{\prime}\left(\lambda_{0}\right)\right|<1 .
$$

Полагая $\lambda_{0}^{(p)}=\lambda_{0}$ и $\lambda_{0}^{(q)}=\lambda_{0}^{(0)}$, получаем

$$
\Phi\left(\lambda_{0}^{(0)}\right)-\alpha\left|\lambda_{0}-\lambda_{0}^{(0)}\right| \leqslant \Phi\left(\lambda_{0}\right) \leqslant \Phi\left(\lambda_{0}^{(0)}\right)+\alpha\left|\lambda_{0}-\lambda_{0}^{(0)}\right| .
$$

С учётом формул (5)-(9) имеем

$$
\Phi\left(\lambda_{0}^{(0)}\right)=\lambda_{0}^{(0)}-c\left[\varphi\left(\lambda_{0}^{(0)}\right)-\lambda_{0}^{(0)}\right]=
$$




$$
=\left\{\begin{array}{cc}
\lambda_{0}^{(0)}+(1-M) \rho, & \text { если } M<1 \\
\lambda_{0}^{(0)}+\operatorname{sign}^{2}\left[1-\varphi^{\prime}\left(\lambda_{0}\right)\right] \frac{(1-\alpha)(1+M)}{1+M} \rho, & \text { если } M>1 .
\end{array}\right.
$$

Отсюда получаем

$$
\Phi\left(\lambda_{0}^{(0)}\right)=\lambda_{0}^{(0)}+(1-\alpha) \rho .
$$

Тогда, используя правую часть неравенства (10), при $0<\alpha<1$ можно получить

$$
\Phi\left(\lambda_{0}\right) \leqslant \Phi\left(\lambda_{0}^{(0)}\right)+\alpha\left(\lambda_{0}-\lambda_{0}^{(0)}\right)=\lambda_{0}^{(0)}+\rho-\alpha\left(\lambda_{0}^{(0)}+\rho-\lambda_{0}\right) \leqslant \lambda_{0}^{(0)}+\rho .
$$

Так как $0 \leqslant \lambda_{0}^{(1)}-\lambda_{0}^{(0)}=\Phi\left(\lambda_{0}^{(0)}\right)-\lambda_{0}^{(0)}=(1-\alpha) \rho<\rho$, то очевидно выполнение следующих неравенств: $\lambda_{0}^{(0)} \leqslant \lambda_{0}^{(0)}+\rho$ и $\lambda_{0}^{(0)} \leqslant \lambda_{0}^{(1)} \leqslant \lambda_{0}^{(0)}+\rho$.

Методом математической индукции покажем, что неравенство

$$
\lambda_{0}^{(0)} \leqslant \lambda_{0}^{(p)} \leqslant \lambda_{0}^{(0)}+\rho
$$

выполняется при любом $p=0,1,2, \ldots$, то есть последовательность $\left\{\lambda_{0}^{(p)}\right\}_{p=0}^{\infty}$ ограничена сверху и снизу.

Предположим, что неравенство (13) выполняется при $p=n-1$. Докажем, что оно будет выполняться и при $p=n$.

Используя левую часть неравенства (10) при $\lambda_{0}=\lambda_{0}^{(n-1)}$, с учётом (11) получаем

$$
\begin{aligned}
\lambda_{0}^{(n)}=\Phi\left(\lambda_{0}^{(n-1)}\right) \geqslant \Phi\left(\lambda_{0}^{(0)}\right)-\alpha\left(\lambda_{0}^{(n-1)}-\lambda_{0}^{(0)}\right) & \geqslant \\
& \geqslant \lambda_{0}^{(0)}+(1-\alpha) \rho-\alpha\left(\lambda_{0}^{(n-1)}-\lambda_{0}^{(0)}\right) .
\end{aligned}
$$

Рассмотрим несколько случаев, соответствующих различным значениям $M$ :

1) пусть $0<M<1 / 2$ и $\lambda_{0}^{(0)} \leqslant \lambda_{0}^{(n-1)} \leqslant \lambda_{0}^{(0)}+\rho$; отсюда $0 \leqslant \lambda_{0}^{(n-1)}-\lambda_{0}^{(0)} \leqslant$ $\leqslant \rho$; тогда из (14) имеем $\lambda_{0}^{(n)} \geqslant \lambda_{0}^{(0)}+(1-\alpha) \rho-\alpha \rho=\lambda_{0}^{(0)}+(1-2 \alpha) \rho=$ $=\lambda_{0}^{(0)}+(1-2 M) \rho \geqslant \lambda_{0}^{(0)}$, так как $1-2 M \geqslant 0$ при $0<M<1 / 2$;

2) пусть $1 / 2 \leqslant M<1$ и $\lambda_{0}^{(0)} \leqslant \lambda_{0}^{(n-1)} \leqslant \lambda_{0}^{(0)}+\frac{1-M}{M} \rho \leqslant \lambda_{0}^{(0)}+\rho$ (или $\left.0 \leqslant \lambda_{0}^{(n-1)}-\lambda_{0}^{(0)} \leqslant \frac{1-M}{M} \rho\right)$; тогда с учётом $\alpha=M$ из (14) получаем $\lambda_{0}^{(n)} \geqslant \lambda_{0}^{(0)}+(1-M) \rho-M \frac{1-M}{M} \rho=\lambda_{0}^{(0)}$;

3) пусть $1 / 2 \leqslant M<1$ и $\lambda_{0}^{(0)}+\frac{1-M}{M} \rho \leqslant \lambda_{0}^{(n-1)} \leqslant \lambda_{0}^{(0)}+\rho$ (или $\frac{1-M}{M} \rho \leqslant$ $\left.\lambda_{0}^{(n-1)}-\lambda_{0}^{(0)} \leqslant \rho\right)$, тогда имеем

$$
\begin{gathered}
\left|\lambda_{0}^{(n)}-\lambda_{0}^{(n-1)}\right|=\left|\Phi\left(\lambda_{0}^{(n-1)}\right)-\Phi\left(\lambda_{0}^{(n-2)}\right)\right| \leqslant \alpha\left|\lambda_{0}^{(n-1)}-\lambda_{0}^{(n-2)}\right| \leqslant \\
\leqslant\left|\Phi\left(\lambda_{0}^{(n-2)}\right)-\Phi\left(\lambda_{0}^{(n-3)}\right)\right| \leqslant \alpha^{2}\left|\lambda_{0}^{(n-2)}-\lambda_{0}^{(n-3)}\right| \leqslant \ldots \leqslant \\
\leqslant \alpha^{n-1}\left|\lambda_{0}^{(1)}-\lambda_{0}^{(0)}\right|
\end{gathered}
$$

отсюда для $0<\alpha<1$ с учётом равенства (11) получаем

$$
\left|\lambda_{0}^{(n)}-\lambda_{0}^{(n-1)}\right|=\alpha^{n-1}\left|\Phi\left(\lambda_{0}^{(0)}\right)-\lambda_{0}^{(0)}\right|<\alpha^{-1}(1-\alpha) \rho ;
$$


тогда при $\alpha=M$ имеем $\left|\lambda_{0}^{(n)}-\lambda_{0}^{(n-1)}\right|<\frac{1-M}{M} \rho \leqslant \lambda_{0}^{(n-1)}-\lambda_{0}^{(0)}$; очевидно, что $\lambda_{0}^{(n)}-\lambda_{0}^{(n-1)} \geqslant-\left|\lambda_{0}^{(n)}-\lambda_{0}^{(n-1)}\right|$; отсюда $\lambda_{0}^{(n)}-\lambda_{0}^{(n-1)}>-\lambda_{0}^{(n-1)}+\lambda_{0}^{(0)}$ или $\lambda_{0}^{(n)}>\lambda_{0}^{(0)}$.

Рассмотрим теперь случаи, когда $M>1$, при этом $\alpha=\frac{1+M-m}{1+M}<1$ :

4) пусть $\frac{1+M}{2} \leqslant m \leqslant M$ и $\lambda_{0}^{(0)} \leqslant \lambda_{0}^{(n-1)} \leqslant \lambda_{0}^{(0)}+\rho$, отсюда $0 \leqslant \lambda_{0}^{(n-1)}-$ $-\lambda_{0}^{(0)} \leqslant \rho$; из $(14)$ следует

$$
\begin{aligned}
\lambda_{0}^{(n)} & \geqslant \lambda_{0}^{(0)}+\left(1-1+\frac{m}{1+M}\right) \rho-\frac{1+M-m}{1+M}\left(\lambda_{0}^{(n-1)}-\lambda_{0}^{(0)}\right) \geqslant \\
& \geqslant \lambda_{0}^{(0)}+\frac{m}{1+M} \rho-\frac{1+M-m}{1+M} \rho=\lambda_{0}^{(0)}+\left(\frac{2 m}{1+M}-1\right) \rho \geqslant \lambda_{0}^{(0)},
\end{aligned}
$$

так как $\frac{2 m}{1+M}-1 \geqslant 0$ при $\frac{1+M}{2} \leqslant m \leqslant M$;

5) пусть $0<m<\frac{1+M}{2}$, при этом $\frac{m}{1+M-m} \rho<\rho$; рассмотрим случай, когда $\lambda_{0}^{(0)} \leqslant \lambda_{0}^{(n-1)} \leqslant \lambda_{0}^{(0)}+\frac{m}{1+M-m} \rho \leqslant \lambda_{0}^{(0)}+\rho$; отсюда получаем $0 \leqslant \lambda_{0}^{(n-1)}$ $-\lambda_{0}^{(0)} \leqslant \frac{m}{1+M-m} \rho$; из (14) имеем

$$
\begin{aligned}
\lambda_{0}^{(n)} \geqslant \lambda_{0}^{(0)}+(1 & \left.-1+\frac{m}{1+M}\right) \rho-\frac{1+M-m}{1+M}\left(\lambda_{0}^{(n-1)}-\lambda_{0}^{(0)}\right) \geqslant \\
& \geqslant \lambda_{0}^{(0)}+\frac{m}{1+M} \rho-\frac{1+M-m}{1+M} \frac{m}{1+M-m} \rho=\lambda_{0}^{(0)}
\end{aligned}
$$

6) пусть $0<m<\frac{1+M}{2}$, при этом $\frac{m}{1+M-m} \rho<\rho$; рассмотрим случай, когда $\lambda_{0}^{(0)} \leqslant \lambda_{0}^{(0)}+\frac{m}{1+M-m} \rho \leqslant \lambda_{0}^{(n-1)} \leqslant \lambda_{0}^{(0)}+\rho$, то есть $\frac{m}{1+M-m} \rho \leqslant \lambda_{0}^{(n-1)}$ $-\lambda_{0}^{(0)} \leqslant \rho$; в соответствии с (15) имеем $\left|\lambda_{0}^{(n)}-\lambda_{0}^{(n-1)}\right|<\alpha^{-1}(1-\alpha) \rho=$ $=\left(\frac{1}{\alpha}-1\right) \rho=\left(\frac{1+M}{1+M-m}-1\right) \rho=\frac{m}{1+M-m} \rho$, следовательно,

$$
\left|\lambda_{0}^{(n)}-\lambda_{0}^{(n-1)}\right|<\lambda_{0}^{(n-1)}-\lambda_{0}^{(0)}
$$

отсюда, аналогично случаю 3 , получаем $\lambda_{0}^{(n)}-\lambda_{0}^{(n-1)}>-\lambda_{0}^{(n-1)}+\lambda_{0}^{(0)}$ или $\lambda_{0}^{(n)}>\lambda_{0}^{(0)}$.

Таким образом, при выполнении условия $\lambda_{0}^{(n-1)} \geqslant \lambda_{0}^{(0)}$ вытекает левая часть неравенства (13) для $p=n: \lambda_{0}^{(n)} \geqslant \lambda_{0}^{(0)}$, то есть последовательность $\left\{\lambda_{0}^{(p)}\right\}_{p=0}^{\infty}$ ограничена снизу.

Пусть правая часть неравенства (13) выполняется при $p=n-1: \lambda_{0}^{(n-1)} \leqslant$ $\leqslant \lambda_{0}^{(0)}+\rho$. Тогда с учётом (12) при $\lambda_{0}=\lambda_{0}^{(n-1)}$ имеем $\lambda_{0}^{(n)}=\Phi\left(\lambda_{0}^{(n-1)}\right) \leqslant \lambda_{0}^{(0)}+\rho$, то есть последовательность $\left\{\lambda_{0}^{(p)}\right\}_{p=0}^{\infty}$ ограничена сверху.

Отсюда по индукции следует, что все члены бесконечной последовательности удовлетворяют неравенству (13), то есть $\lambda_{0}^{(p)} \in\left[\lambda_{0}^{(0)}, \lambda_{0}^{(0)}+\rho\right] \subset\left[\lambda_{0}^{(0)}, \lambda_{0}^{(0)}+\right.$ $+l], p=0,1,2, \ldots$ 
Покажем, что рассматриваемая последовательность $\left\{\lambda_{0}^{(p)}\right\}_{p=0}^{\infty}-$ фундаментальная. Учитывая полученные выше неравенства

$$
\left|\lambda_{0}^{(n)}-\lambda_{0}^{(n-1)}\right| \leqslant \alpha^{n-1}\left|\lambda_{0}^{(1)}-\lambda_{0}^{(0)}\right|, \quad 0<\alpha<1
$$

и соотношение $0 \leqslant \lambda_{0}^{(1)}-\lambda_{0}^{(0)}=(1-\alpha) \rho<\rho \leqslant l$, получаем

$$
\begin{aligned}
\left|\lambda_{0}^{(k+p)}-\lambda_{0}^{(k)}\right| \leqslant \sum_{n=k+1}^{k+p}\left|\lambda_{0}^{(n)}-\lambda_{0}^{(n-1)}\right| \leqslant \sum_{n=k+1}^{k+p} \alpha^{n-1}\left|\lambda_{0}^{(1)}-\lambda_{0}^{(0)}\right| \leqslant & \\
& \leqslant(1-\alpha) \rho \sum_{n=k+1}^{k+p} \alpha^{n-1} \leqslant(1-\alpha) l \alpha^{k} \frac{1-\alpha^{p}}{1-\alpha}<l \alpha^{k}
\end{aligned}
$$

где $k$ и $p$-любые натуральные числа.

Так как $\alpha^{k} \rightarrow 0$ при $k \rightarrow \infty$, последовательность $\left\{\lambda_{0}^{(p)}\right\}_{p=0}^{\infty}$ удовлетворяет критерию Коши и, следовательно, существует $\lim _{p \rightarrow \infty} \lambda_{0}^{(p)}=\bar{\lambda}_{0}$. Причём, если элементы последовательности $\left\{\lambda_{0}^{(p)}\right\}_{p=0}^{\infty}$ принадлежат отрезку $\left[\lambda_{0}^{(0)}, \lambda_{0}^{(0)}+l\right]$, то и $\bar{\lambda}_{0} \in\left[\lambda_{0}^{(0)}, \lambda_{0}^{(0)}+l\right]$.

Таким образом, числовая последовательность $\left\{\lambda_{0}^{(p)}\right\}_{p=0}^{\infty}$, описываемая рекуррентной формулой $(2)$, при выполнении условий теоремы имеет $\lim _{p \rightarrow \infty} \lambda_{0}^{(p)}=$ $=\bar{\lambda}_{0}$, принадлежащий отрезку $\left[\lambda_{0}^{(0)}, \lambda_{0}^{(0)}+l\right]$.

Так как функция $\Phi\left(\lambda_{0}\right)=(1+c) \lambda_{0}-c \varphi\left(\lambda_{0}\right)$ по условию теоремы непрерывна на отрезке $\left[\lambda_{0}^{(0)}, \lambda_{0}^{(0)}+l\right]$, можно перейти к пределу при $p \rightarrow \infty$, осуществив предельный переход справа под знаком функции $\Phi\left(\lambda_{0}^{(p)}\right)$ :

$$
\begin{aligned}
\lim _{p \rightarrow \infty} \lambda_{0}^{(p)}=\lim _{p \rightarrow \infty} \Phi\left(\lambda_{0}^{(p)}\right)=\lim _{p \rightarrow \infty}\left[(1+c) \lambda_{0}^{(p)}-c \varphi\left(\lambda_{0}^{(p)}\right)\right]= \\
=(1+c) \lim _{p \rightarrow \infty} \lambda_{0}^{(p)}-c \varphi\left(\lim _{p \rightarrow \infty} \lambda_{0}^{(p)}\right)=(1+c) \bar{\lambda}_{0}-c \varphi\left(\bar{\lambda}_{0}\right) .
\end{aligned}
$$

Отсюда получаем $\bar{\lambda}_{0}=\bar{\lambda}_{0}+c\left(\bar{\lambda}_{0}-\varphi\left(\bar{\lambda}_{0}\right)\right)$. При $c \neq 0$ имеем, что $\bar{\lambda}_{0}=\varphi\left(\bar{\lambda}_{0}\right)$, то есть предельное значение $\bar{\lambda}_{0} \in\left[\lambda_{0}^{(0)}, \lambda_{0}^{(0)}+l\right]$ является корнем уравнения $\lambda_{0}=\varphi\left(\lambda_{0}\right)$.

Пусть $\overline{\bar{\lambda}}_{0} \in\left[\lambda_{0}^{(0)}, \lambda_{0}^{(0)}+l\right]$ также является решением уравнения $\lambda_{0}=\varphi\left(\lambda_{0}\right)$, то есть $\overline{\bar{\lambda}}_{0}=\varphi\left(\overline{\bar{\lambda}}_{0}\right)$. Так как функция $\varphi\left(\lambda_{0}\right)$ непрерывно дифференцируема на отрезке $\left[\lambda_{0}^{(0)}, \lambda_{0}^{(0)}+l\right]$, она удовлетворяет на $\left[\lambda_{0}^{(0)}, \lambda_{0}^{(0)}+l\right]$ условию Липшица с постоянной $M=\max _{\left[\lambda_{0}^{(0)}, \lambda_{0}^{(0)}+l\right]}\left[\varphi^{\prime}\left(\lambda_{0}\right)\right] \neq 1$. Следовательно, имеет место неравенство $\left|\bar{\lambda}_{0}-\overline{\bar{\lambda}}_{0}\right|=\left|\varphi\left(\bar{\lambda}_{0}\right)-\varphi\left(\overline{\bar{\lambda}}_{0}\right)\right| \leqslant M\left|\bar{\lambda}_{0}-\overline{\bar{\lambda}}_{0}\right|$. Отсюда получаем $\left|\bar{\lambda}_{0}-\overline{\bar{\lambda}}_{0}\right|(1-M) \leqslant 0$. Так как по условию теоремы $M \neq 1$, неравенство выполняется только при $\bar{\lambda}_{0}=\overline{\bar{\lambda}}_{0}$. Единственность решения уравнения $\lambda_{0}=\varphi\left(\lambda_{0}\right)$ на отрезке $\left[\lambda_{0}^{(0)}, \lambda_{0}^{(0)}+l\right]$ установлена. 
СледствиЕ. Априорная и апостериорная оченки погрешности $n$-ного приближения описываются соответственно следующими формулами:

$$
\begin{gathered}
\left|\bar{\lambda}_{0}-\lambda_{0}^{(n)}\right| \leqslant \rho M^{n}, \\
\left|\bar{\lambda}_{0}-\lambda_{0}^{(n)}\right| \leqslant \frac{M}{1-M}\left|\lambda_{0}^{(n)}-\lambda_{0}^{(n-1)}\right|
\end{gathered}
$$

при $0<M<1$ и $\rho=\frac{\varphi\left(\lambda_{0}^{(0)}\right)-\lambda_{0}^{(0)}}{1-M} \leqslant l$ или формулами

$$
\begin{gathered}
\left|\bar{\lambda}_{0}-\lambda_{0}^{(n)}\right| \leqslant\left(1-\frac{m}{1+M}\right)^{n} \rho, \\
\left|\bar{\lambda}_{0}-\lambda_{0}^{(n)}\right| \leqslant \frac{1+M-m}{1+M}\left|\lambda_{0}^{(n)}-\lambda_{0}^{(n-1)}\right|
\end{gathered}
$$

$n p u\left\{\begin{array}{l}M>1, \\ \left|\varphi^{\prime}\left(\lambda_{0}\right)-1\right| \geqslant m, \quad u \rho=\frac{\operatorname{sign}\left[1-\varphi^{\prime}\left(\lambda_{0}\right)\right]\left[\varphi\left(\lambda_{0}^{(0)}\right)-\lambda_{0}^{(0)}\right]}{m} \leqslant l . \\ 0<m<M\end{array}\right.$

Доказательство. Действительно, с учётом равенств $\bar{\lambda}_{0}=\Phi\left(\bar{\lambda}_{0}\right)$ и $\lambda_{0}^{(n)}=\Phi\left(\lambda_{0}^{(n-1)}\right)$ и непрерывной дифференцируемости функции $\Phi\left(\lambda_{0}\right)$ имеем

$$
\begin{aligned}
\left|\bar{\lambda}_{0}-\lambda_{0}^{(n)}\right|= & \left|\Phi\left(\bar{\lambda}_{0}\right)-\Phi\left(\lambda_{0}^{(n-1)}\right)\right| \leqslant \alpha\left|\bar{\lambda}_{0}-\lambda_{0}^{(n-1)}\right|= \\
& =\alpha\left|\Phi\left(\bar{\lambda}_{0}\right)-\Phi\left(\lambda_{0}^{(n-2)}\right)\right| \leqslant \alpha^{2}\left|\bar{\lambda}_{0}-\lambda_{0}^{(n-2)}\right| \leqslant \ldots \leqslant \alpha^{n}\left|\bar{\lambda}_{0}-\lambda_{0}^{(0)}\right| .
\end{aligned}
$$

Так как $\bar{\lambda}_{0} \in\left[\lambda_{0}^{(0)}, \lambda_{0}^{(0)}+\rho\right]$, можно записать, что $\left|\bar{\lambda}_{0}-\lambda_{0}^{(0)}\right| \leqslant \rho$. Поэтому имеет место неравенство $\left|\bar{\lambda}_{0}-\lambda_{0}^{(n)}\right| \leqslant \alpha^{n} \rho$.

Пусть $0<M<1$ и в соответствии с (7) $\rho=\frac{\varphi\left(\lambda_{0}^{(0)}\right)-\lambda_{0}^{(0)}}{1-M} \leqslant l$. Тогда при $\alpha=M<1$ можно получить формулу априорной оценки погрешности (16).

Пусть $\left\{\begin{array}{l}M>1, \\ \left|\varphi^{\prime}\left(\lambda_{0}\right)-1\right| \geqslant m, \quad \text { и в соответствии с формулами (8) и }(9) \\ 0<m<M\end{array}\right.$

$$
\rho=\frac{\operatorname{sign}\left[1-\varphi^{\prime}\left(\lambda_{0}\right)\right]\left[\varphi\left(\lambda_{0}^{(0)}\right)-\lambda_{0}^{(0)}\right]}{m} \leqslant l .
$$

Для этого случая $\alpha=\frac{1+M-m}{1+M}$ и можно получить формулу априорной оценки погрешности (18).

Для построения формул апостериорной оценки погрешности $n$-ного приближения воспользуемся соотношением

$$
\bar{\lambda}_{0}-\lambda_{0}^{(n-1)}=\lambda_{0}^{(n)}-\lambda_{0}^{(n-1)}+\Phi\left(\bar{\lambda}_{0}\right)-\Phi\left(\lambda_{0}^{(n-1)}\right),
$$

из которого с учётом условия Липшица находим

$$
\left|\bar{\lambda}_{0}-\lambda_{0}^{(n-1)}\right| \leqslant\left|\lambda_{0}^{(n)}-\lambda_{0}^{(n-1)}\right|+\alpha\left|\bar{\lambda}_{0}-\lambda_{0}^{(n-1)}\right|
$$


или

$$
\left|\bar{\lambda}_{0}-\lambda_{0}^{(n-1)}\right| \leqslant \frac{1}{1-\alpha}\left|\lambda_{0}^{(n)}-\lambda_{0}^{(n-1)}\right|
$$

Так как $\left|\bar{\lambda}_{0}-\lambda_{0}^{(n)}\right| \leqslant \alpha\left|\bar{\lambda}_{0}-\lambda_{0}^{(n-1)}\right|$, можно записать, что

$$
\left|\bar{\lambda}_{0}-\lambda_{0}^{(n)}\right| \leqslant \frac{\alpha}{1-\alpha}\left|\lambda_{0}^{(n)}-\lambda_{0}^{(n-1)}\right|
$$

Пусть $0<M<1$. Тогда при $\alpha=M<1$ можно получить формулу апостериорной оценки погрешности (17).

Пусть $\left\{\begin{array}{l}M>1, \\ \left|\varphi^{\prime}\left(\lambda_{0}\right)-1\right| \geqslant m, \quad \text { Для этого случая } \alpha=\frac{1+M-m}{1+M} \text { и можно полу- } \\ 0<m<M .\end{array}\right.$ чить формулу апостериорной оценки погрешности (19).

Таким образом, рассмотрено достаточное условие сходимости итерационной процедуры уточнения коэффициентов разностного уравнения, предназначенной для повышения точности вычисления (почти в два раза [2]) параметров дифференциального оператора для систем с турбулентным трением. Следует отметить, и это очевидно, что область применения итерационной процедуры, описываемой формулой вида

$$
x_{k}=(1+c) x_{k-1}-c \varphi\left(x_{k-1}\right) \text {, }
$$

в которой $\varphi(x)$ - непрерывно дифференцируемая на отрезке $[a, b]$ функция и $\varphi^{\prime}\left(\lambda_{0}\right) \leqslant M$, не ограничивается только задачей среднеквадратичного оценивания коэффициентов разностного уравнения. Алгоритм вычислений с использованием этой рекуррентной формулы может применяться в различных по физической природе задачах математического моделирования. Эта формула, обобщая при $c=1$ известный метод итераций для значений $M<1$, обеспечивает сходимость итерационной процедуры также для значений $M>1$ за счёт выбора параметра $c$ в соответствии с выражением (5).

Работа выполнена при поддержке Министерства образования и науки РФ (проект РНП 2.1.1/14069).

\section{БИБЛИОГРАФИЧЕСКИЙ СПИСОК}

1. Зотеев В. Е. Параметрическая идентификация диссипативных механических систем на основе разностных уравнений / ред. В. П. Радченко. М.: Машиностроение-1, 2009. 344 с. [Zoteev V. E. Parametric identification of dissipative mechanical systems based on difference equations / ed. V. P. Radchenko. Moscow: Mashinostroenie-1, 2009. 344 pp.]

2. Зотеев B.E., Заусаева М.А., Егорова А.А. Параметрическая идентификация дифференциальных операторов для систем с турбулентным трением на основе разностных уравнений // Вестн. Сам. гос. техн. ун-та. Сер. Физ.-мат. науки, 2010. № 5(21). С. 125133. [Zoteev V. E., Zausaeva M. A., Egorova A. A. Parametric identification of the differential operators for the systems with turbulent friction on the base of finite-difference equations // Vestn. Samar. Gos. Tekhn. Univ. Ser. Fiz.-Mat. Nauki, 2010. Vol.5(21). Pp. 125-133].

Поступила в редакцию 04/IX/2011;

в окончательном варианте - 19/IX/2011. 
MSC: $65 \mathrm{C20} ; 65 \mathrm{P} 40,34 \mathrm{C} 15,37 \mathrm{M05}$

\title{
PROBLEM OF ITERATIVE PROCEDURE CONVERGENCE \\ IN PARAMETRIC IDENTIFICATION OF THE SYSTEMS WITH TURBULENT FRICTION
}

\author{
V. E. Zoteev, M. A. Romanyuk, A.A. Egorova
}

Samara State Technical University, 244, Molodogvardeyskaya st., Samara, 443100, Russia.

E-mail: zoteev-ve@mail.ru

The problem of convergence of iterative procedure for clarification of coefficients of the finite-difference equation in numerical technique for determination of the differential operator parameters for the systems with turbulent friction is considered. Theorem about a sufficient condition of iterative procedure convergence is defined and demonstrated. During theorem proving contracting mapping and fundamental sequence properties are used. As a corollary of the theorem formulas describing prior and posterior estimate of the approximation error are received.

Key words: systems with turbulent friction, parametric identification, finite-difference equations, mean-square estimation.

Original article submitted 04/IX/2011; revision submitted 19/IX/2011.

Vladimir E. Zoteev (Dr. Sci. (Techn.)), Professor, Dept. of Applied Mathematics \& Computer Science. Maria A. Zausaeva, Assistant, Dept. of Applied Mathematics \& Computer Science. Alexandra A. Egorova, Postgraduate Student, Dept. of Applied Mathematics \& Computer Science. 